\title{
A new method for measuring the imaginary part of the atmospheric refractive index structure parameter in the urban surface layer
}

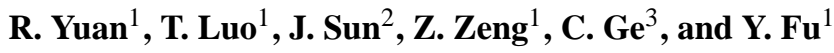 \\ ${ }^{1}$ Key Laboratory of the Atmospheric Composition and Optical Radiation, CAS, School of Earth and Space Sciences, \\ University of Science and Technology of China, Anhui, 230026, China \\ ${ }^{2}$ School of Atmospheric Sciences, Nanjing University, Jiangsu, 210023, China \\ ${ }^{3}$ Optoelectronic Engineering Laboratory, Army Officers Academy, PLA, Anhui, 230031, China
}

Correspondence to: R. Yuan (rmyuan@ustc.edu.cn) and T. Luo (luotao@ustc.edu.cn)

Received: 20 July 2014 - Published in Atmos. Chem. Phys. Discuss.: 20 August 2014

Revised: 25 December 2014 - Accepted: 8 February 2015 - Published: 6 March 2015

\begin{abstract}
The atmospheric refractive index consists of both real and imaginary parts. The intensity of refractive index fluctuations is generally expressed as the refractive index structure parameter, with the real part reflecting the strength of atmospheric turbulence and the imaginary part reflecting absorption in the light path. A large aperture scintillometer (LAS) is often used to measure the structure parameter of the real part of the atmospheric refractive index, from which the sensible and latent heat fluxes can further be obtained, whereas the influence of the imaginary part is ignored or considered noise. In this theoretical analysis study, the relationship between logarithmic light intensity variance and the atmospheric refractive index structure parameter (ARISP), as well as that between the logarithmic light intensity structure function and the ARISP, is derived. Additionally, a simple expression for the imaginary part of the ARISP is obtained which can be conveniently used to determine the imaginary part of the ARISP from LAS measurements. Moreover, these relationships provide a new method for estimating the outer scale of turbulence. Light propagation experiments were performed in the urban surface layer, from which the imaginary part of the ARISP was calculated. The experimental results showed good agreement with the presented theory. The results also suggest that the imaginary part of the ARISP exhibits a different diurnal variation from that of the real part. For the wavelength of light used $(0.62 \mu \mathrm{m})$, the variation of the imaginary part of the ARISP is related to both the turbulent transport process and the spatial distribution characteristics of aerosols.
\end{abstract}

\section{Introduction}

A line-of-sight light wave propagating in the atmosphere undergoes scattering by atmospheric turbulence, gas molecules and aerosol particles, as well as absorption by gas molecules and aerosol particles. After the light propagates some distance, fluctuations in the light intensity are related not only to the inhomogeneous scattering by turbulence and particles, but also to the absorption occurring along the light path. According to optical propagation theory (Rao, 2012; Tatarskii, 1961), the scattering is associated with the real part of the atmospheric refractive index, and the absorption is associated with the imaginary part. Based on the relationship between the light intensity fluctuations in the receiving plane after propagation for some distance and the fluctuations of the real part of the atmospheric refractive index (Clifford, 1971; De Bruin and Evans, 2012; Wang et al., 1978), the real part of the atmospheric refractive index structure parameter (ARISP) can be deduced. Under the free-convection condition, the real part of the ARISP is related to the turbulent transport of temperature and water vapour (Wyngaard et al., 1971), which allows a large-aperture scintillometer (LAS) to determine the sensible heat flux and latent heat flux by measuring light intensity fluctuations (Andreas, 1989). Although absorption inevitably occurs when an electromagnetic wave propagates in the atmosphere, certain mathematical methods can be used to remove the contribution of absorption to scintillation under weak atmospheric absorption conditions (Solignac et al., 2012). However, it is important to understand the role of absorption in the observed scintillation un- 
der strong atmospheric absorption conditions, such as a polluted atmospheric boundary layer or selected atmospheric absorption regions. This study aims to develop a theoretical framework to analyse the contribution of absorption to scintillation, which can be used to derive the imaginary part of the ARISP in the urban atmospheric boundary layer from scintillation measurements.

As early as 1983, Filho et al. (1983) measured the scintillation spectra of a microwave propagating over a distance of $4.1 \mathrm{~km}$ in central London (Filho et al., 1983). The frequency of the microwave used was off resonance from the $60 \mathrm{GHz}$ oxygen absorption peak, and the wavelength $(5.4 \mathrm{~mm})$ was much larger than the size of aerosol particles. Analysis showed that the lower corner frequency derived from the scintillation spectra using the method proposed by Ott and Thompson Jr. (1978) is very close to the $\mathrm{O}_{2}$ absorption region in which the scintillation is enhanced. This result suggests that the effect of absorption can be determined from scintillation measurements. Nieveen et al. (1998) obtained a value of approximately $4.1 \times 10^{-24} \mathrm{~m}^{-2 / 3}$ for the imaginary part of the ARISP at a pasture site based on scintillation data collected over a distance of $248 \mathrm{~m}$ for a wavelength of $0.940 \mu \mathrm{m}$, which lies inside an absorption band of water vapour. However, their experiments did not measure absorption by aerosol particles. In those studies, the imaginary part of the ARISP was obtained by the lower corner frequency in the spectral densities of light intensity fluctuations. However, it is difficult to objectively identify the lower corner frequency due to the variation of the spectral density at low frequencies.

Limited research has been conducted on the contribution of aerosol absorption to the intensity fluctuations of light waves. One possible reason is that aerosol concentrations are very low in several areas, making the contribution of aerosol absorption negligible. However, many developing countries are suffering from increasing aerosol pollution containing a high fraction of soot. For example, several cities in China are experiencing increasingly serious fog and haze conditions, which strongly affect visibility and radiation levels (Ding and Liu, 2014). Soot aerosols have strong and broad absorption bands. A few studies have been performed to measure the mean imaginary part of the aerosol refractive index (Raut and Chazette, 2008; Zhang et al., 2013), the results of which indicate that the temporal and spatial distributions of the imaginary refractive index are highly variable. The inhomogeneity of the imaginary refractive index can be described by the imaginary part of the ARISP. That is, an increase in the imaginary part of the ARISP suggests an increase in the inhomogeneity of the imaginary refractive index and vice versa. Therefore, knowledge of the imaginary part of the ARISP can be used to further understand the temporal and spatial distributions of the imaginary refractive index and aerosol transport.

Based on the proposed theoretical framework and the LAS experiments performed, which employ visible light in the urban surface layer, herein we outline the development of a new objective method for obtaining the imaginary part of the ARISP by combining light fluctuation variances and structure functions, which include the contribution of the imaginary refractive index. The experiments were performed in Hefei, China, and the results show that the imaginary part of the ARISP can be reliably derived by this method.

Section 2 presents the theoretical framework, and the experiment is described in Sect. 3. The experimental results are presented in Sect. 4, which show that the proposed approach is capable of providing the characteristics of the imaginary part of the ARISP in the urban boundary layer. Finally, a brief conclusion is presented.

\section{Methodology based on theoretical analysis}

\subsection{Relationship between log-intensity variance and the imaginary part of the ARISP}

For a planar or spherical wave in a slowly varying turbulence field, the two-dimensional log-intensity spectrum is (Filho et al., 1983)

$$
\begin{aligned}
& F_{\ln } I(\kappa, L)=8 \pi \eta^{2} \int_{0}^{L}\left\{\Phi_{n, \operatorname{Re}}(\kappa) \sin ^{2}(\theta)\right. \\
& \left.+\Phi_{n, \mathrm{Im}}(\kappa) \cos ^{2}(\theta)+\Phi_{n, \mathrm{IR}}(\kappa) \sin (2 \theta)\right\} \mathrm{d} x,
\end{aligned}
$$

where $\theta$ is $\kappa^{2} x(L-x) / 2 \eta L$ for a spherical wave or $\kappa^{2}(L-$ $x) / 2 \eta$ for a plane wave (in this study, only the spherical wave case was considered), $\kappa$ is the wave number of the twodimensional $\log$-intensity spectrum, $\eta$ is the wave number of the spherical wave, $x$ is the position of the propagating wave and $L$ is the length of propagation path. Additionally, $\Phi_{n}$ is the spectrum of the refractive index, where the subscript $n$ denotes the refractive index, the subscripts Re and Im denote the real and the imaginary parts of the refractive index, respectively, and the subscript IR denotes the correlation between the real and imaginary parts. In the following analysis, it is assumed that the fluctuations in the real and imaginary parts of the refractive index are not correlated (Filho et al., 1983); therefore, the joint term can be neglected.

In the atmospheric window wave used for propagation, the absorption contribution is due to aerosols. Observations over the urban surface under unstable atmospheric stratification showed that aerosol concentration fluctuations exhibit a $-2 / 3$ power law dependence and that the aerosol concentration-velocity co-spectra follow a $-4 / 3$ power law dependence (Mårtensson et al., 2006; Vogt et al., 2011). We can therefore simply assume that the absorption media and temperature in the atmosphere are conservative and passive scalars, with their sources at the surface, and ignore the force on the turbulence. Under this assumption, $\Phi_{n, \operatorname{Re}}$ and $\Phi_{n, \mathrm{Im}}$ have the same form. The widely used von Karman spectral form for $\Phi_{n, \operatorname{Re}}$ and $\Phi_{n, \mathrm{Im}}$ is adopted in this study (Andrews 
and Phillips, 2005), which can be expressed as follows:

$\Phi_{n, \operatorname{Re}}(\kappa)=0.033 C_{n, \operatorname{Re}}^{2}\left(\kappa^{2}+\frac{1}{L_{0}^{2}}\right)^{-\frac{11}{6}} e^{-\frac{\kappa^{2} l_{0}^{2}}{5.92^{2}}}$,

$\Phi_{n, \operatorname{Im}}(\kappa)=0.033 C_{n, \operatorname{Im}}^{2}\left(\kappa^{2}+\frac{1}{L_{0}^{2}}\right)^{-\frac{11}{6}} e^{-\frac{\kappa^{2} l_{0}^{2}}{5.92^{2}}}$.

Here, $C_{n, \mathrm{Re}}^{2}$ and $C_{n, \mathrm{Im}}^{2}$ are the real and imaginary parts of the ARISP, respectively, $L_{0}$ is the outer scale of turbulence and $l_{0}$ is the inner scale of turbulence. Although some measurements have revealed that the turbulence often shows anisotropic characteristics (Consortini et al., 1970; Yuan et al., 2014), the isotropic turbulence assumption will still be used in this paper due to the rather high measurement level (this will be further discussed in Sect. 3.1).

Assuming isotropic turbulence, the one-dimensional spatial spectrum can be derived by integrating Eq. (1). It can then be converted to a temporal spectrum according to the Taylor frozen hypothesis. Considering aperture-smoothing effects, the temporal spectrum caused by the real part of the ARISP can be expressed as (Nieveen et al., 1998; Clifford, 1971)

$$
\begin{aligned}
& W_{\ln I, \operatorname{Re}}(f)=64 \pi^{2} \eta^{2} \int_{0}^{L} \mathrm{~d} x \int_{2 \pi f / v}^{\infty} \\
& \Phi_{n, \operatorname{Re}}(\kappa) \sin ^{2}\left[\frac{\kappa^{2} x(L-x)}{2 \eta L}\right]\left[(\kappa v)^{2}-(2 \pi f)^{2}\right]^{-1 / 2} \\
& \cdot\left[\frac{2 J_{1}\left(\frac{D_{\mathrm{r}} \kappa x}{2 L}\right)}{D_{\mathrm{r}} \kappa x / 2 L}\right]^{2}\left[\frac{2 J_{1}\left(\frac{D_{\mathrm{t}} \kappa(L-x)}{2 L}\right)}{D_{\mathrm{t}} \kappa(L-x) / 2 L}\right]^{2} \kappa \mathrm{d} \kappa .
\end{aligned}
$$

Here, $D_{\mathrm{t}}$ is the transmitting aperture diameter, $D_{\mathrm{r}}$ is the receiving aperture diameter $\left(D_{\mathrm{t}}\right.$ and $D_{\mathrm{r}}$ are usually identical for a LAS), $v$ is the transverse wind speed and $J_{1}$ is the firstorder Bessel function.

Similarly, the temporal spectrum due to the imaginary part of the ARISP can be derived and expressed as (Nieveen et al., 1998; Clifford, 1971)

$$
\begin{aligned}
& W_{\ln I, \operatorname{Im}}(f)=64 \pi^{2} \eta^{2} \int_{0}^{L} \mathrm{~d} x \int_{2 \pi f / v}^{\infty} \\
& \Phi_{n, \operatorname{Im}}(\kappa) \cos ^{2}\left[\frac{\kappa^{2} x(L-x)}{2 \eta L}\right]\left[(\kappa v)^{2}-(2 \pi f)^{2}\right]^{-1 / 2} \\
& \cdot\left[\frac{2 J_{1}\left(\frac{D_{\mathrm{r}} \kappa x}{2 L}\right)}{D_{\mathrm{r}} \kappa x / 2 L}\right]^{2}\left[\frac{2 J_{1}\left(\frac{D_{\mathrm{t}} \kappa(L-x)}{2 L}\right)}{D_{\mathrm{t}} \kappa(L-x) / 2 L}\right]^{2} \kappa \mathrm{d} \kappa .
\end{aligned}
$$

Numerical integration of Eqs. (2) and (3) yields the logintensity spectrum for the real and the imaginary parts of the ARISP, respectively.

Figure 1 shows an example of the temporal spectrum of the log-intensity calculated from Eqs. (2) and (3) with parameters of $C_{n, \operatorname{Re}}^{2}=9.5 \times 10^{-15} \mathrm{~m}^{-2 / 3}, C_{n, \mathrm{Im}}^{2}=4.0 \times$ $10^{-24} \mathrm{~m}^{-2 / 3}, L_{0}=27.1 \mathrm{~m}, L=960 \mathrm{~m}, D_{\mathrm{t}}=D_{\mathrm{r}}=0.18 \mathrm{~m}$

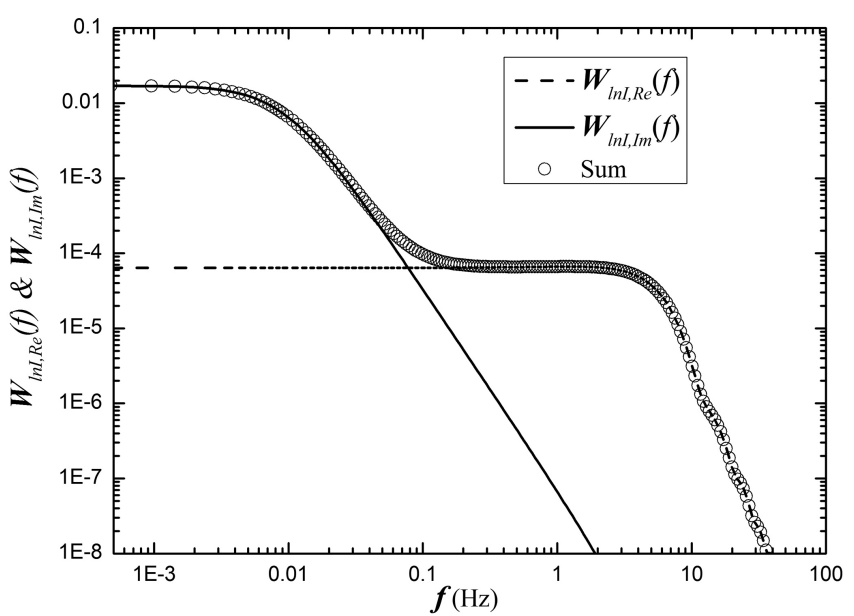

Figure 1. The theoretical temporal spectral densities $\left(W_{\ln I, \operatorname{Re}}(f)\right.$ and $\left.W_{\ln I, \operatorname{Im}}(f)\right)$ of the logarithmic light intensity fluctuations due to the real and imaginary parts of the ARISP (see text for the parameter values used in the calculation).

and $v=1.3 \mathrm{~m} \mathrm{~s}^{-1}$ (the method used to obtain $C_{n, \mathrm{Re}}^{2}, C_{n, \mathrm{Im}}^{2}$ and $L_{0}$ will be described in Sect. 3.3). The dashed and solid lines in Fig. 1 represent the temporal spectra of the $\log$ intensity that are related to the real and imaginary parts of the ARISP, respectively. The sum of the contributions from the real and imaginary parts are plotted as circles, and these values correspond to the measured spectrum of the log-intensity obtained from LAS experiments. At low frequencies, the contribution of the imaginary part of the refractive index to the measured spectrum (i.e., the sum in Fig. 1) is dominant, whereas that of the real part is negligibly small. The frequency distributions of the spectral densities make it possible to assume that the real and imaginary parts are independent. This characteristic allows the contribution of the imaginary part of the refractive index to be determined from the density spectrum obtained from LAS measurements.

As shown in Fig. 1, the temporal spectrum of the logintensity due to the real part of the refractive index reaches a plateau at frequencies below $3 \mathrm{~Hz}$. This property suggests that the spectral density of the plateau region $\left(\mathrm{WP}_{\ln I, \mathrm{Re}}\right)$ can be numerically calculated as

$\mathrm{WP}_{\ln I, \operatorname{Re}}=1.04 L^{3} D_{\mathrm{t}}^{-2 / 3} D_{\mathrm{r}}^{-2 / 3} C_{n, \operatorname{Re}}^{2} v^{-1}$.

The variance of the log-intensity can be derived from the spectrum. As shown in Fig. 1, the contribution of the real part of the refractive index to the spectrum primarily occurs at higher frequencies, whereas that of the imaginary part mainly occurs at lower frequencies. These characteristics imply that the log-intensity variances caused by the real and imaginary parts are independent. Thus, the log-intensity variances corresponding to the real and imaginary parts can be determined separately at high frequencies and low frequencies from the LAS measurements. 
Integrating Eq. (2) yields an expression for the logintensity variance caused by the real part of the ARISP (Wang et al., 1978):

$\sigma_{\ln I, \operatorname{Re}}^{2}=0.89 C_{n, \operatorname{Re}}^{2} L^{3} D_{\mathrm{t}}^{-7 / 6} D_{\mathrm{r}}^{-7 / 6}$.

In Eq. (5), $L, D_{\mathrm{t}}$ and $D_{\mathrm{r}}$ are constants; thus, $\sigma_{\ln I, \operatorname{Re}}^{2}$ depends only on the real part of the ARISP, $C_{n, \mathrm{Re}}^{2}$. This equation can be alternatively expressed as

$C_{n, \operatorname{Re}}^{2}=1.12 \sigma_{\ln I, \operatorname{Re}}^{2} L^{-3} D_{\mathrm{t}}^{7 / 6} D_{\mathrm{r}}^{7 / 6}$.

Equation (6) is the expression commonly used to determine the real part of the ARISP from LAS measurements (Wang et al., 1978).

Combining Eqs. (4) and (5), the transverse velocity can be written as

$v=\frac{1.16 D_{\mathrm{t}}^{1 / 2} D_{\mathrm{r}}^{1 / 2} \sigma_{\ln I, \mathrm{Re}}^{2}}{\mathrm{WP}_{\ln I, \operatorname{Re}}}$.

Similarly, integrating Eq. (3) yields the log-intensity variance due to the imaginary part of the ARISP:

$\sigma_{\ln I, \operatorname{Im}}^{2}=2.95 C_{n, \operatorname{Im}}^{2} \eta^{2} L_{0}^{5 / 3} L$

The form of Eq. (8) reveals that $\sigma_{\ln I, \text { Im }}^{2}$ depends not only on the imaginary part of the ARISP $\left(C_{n, \mathrm{Im}}^{2}\right)$ but also on the outer scale of turbulence $\left(L_{0}\right)$. The relationship is a new one regarding the characteristics of absorption effects on light propagation, and it implies that $C_{n, \operatorname{Im}}^{2}$ and $L_{0}$ can be derived from LAS measurements when additional information can be obtained, as will be demonstrated in the next section.

\subsection{Relationship between the structure function and the imaginary part of the ARISP}

The two-point $\log$-intensity correlation moment $\left(B_{\ln I(\rho)}\right)$ with a distance $\rho$ is defined as (Ishimaru, 1997)

$B_{\ln I}(\rho)=2 \pi \int_{0}^{\infty} F_{\ln I}(\kappa, L) J_{0}(\kappa \rho) \kappa \mathrm{d} \kappa$.

In the above expression, $J_{0}$ is the zero-order Bessel function.

According to the relationship between the correlation moment and structure function, the structure function $\left(D_{\ln } I(\rho)\right)$ can be expressed as

$D_{\ln I}(\rho)=4 \pi \int_{0}^{\infty} F_{\ln I}(\kappa, L)\left[1-J_{0}(\kappa \rho)\right] \kappa \mathrm{d} \kappa$.

Upon invoking the aperture smoothing effect, the logintensity structure function due to the real part of the ARISP becomes

$D_{\ln I, \operatorname{Re}}(\rho)=32 \pi^{2} \eta^{2} \int_{0}^{L} \mathrm{~d} x \int_{0}^{\infty}$

$\Phi_{n, \operatorname{Re}} \sin ^{2}(\theta)\left[1-J_{0}(\kappa \rho)\right]\left[\frac{2 J_{1}\left(\frac{D_{\mathrm{r}} \kappa x}{2 L}\right)}{D_{\mathrm{r}} \kappa x / 2 L}\right]^{2}$

$\left[\frac{2 J_{1}\left(\frac{D_{\mathrm{t}} \kappa(L-x)}{2 L}\right)}{D_{\mathrm{t}} \kappa(L-x) / 2 L}\right]^{2} \kappa \mathrm{d} \kappa$.

With further derivation steps, the following simplified relationships are valid under the various conditions stated:

$D_{\ln I, \operatorname{Re}}(\rho)=14.8 C_{n, \operatorname{Re}}^{2} L^{3} D_{\mathrm{t}}^{-13 / 6} D_{\mathrm{r}}^{-13 / 6} \rho^{2}\left(\rho \ll D_{\mathrm{t}}\left(\right.\right.$ or $\left.\left.D_{\mathrm{r}}\right)\right)$,

$D_{\ln I, \operatorname{Re}}(\rho)=4.3 C_{n, \operatorname{Re}}^{2} L^{3} D_{\mathrm{t}}^{-11 / 6} D_{\mathrm{r}}^{-11 / 6} \rho^{4 / 3}\left(\rho<D_{\mathrm{t}}\left(\right.\right.$ or $\left.\left.D_{\mathrm{r}}\right)\right)$,

$D_{\ln I, \operatorname{Re}}(\rho)=1.78 C_{n, \operatorname{Re}}^{2} L^{3} D_{\mathrm{t}}^{-7 / 6} D_{\mathrm{r}}^{-7 / 6}\left(\rho \geq D_{\mathrm{t}}\left(\right.\right.$ or $\left.\left.D_{\mathrm{r}}\right)\right)$.

Equation (12c) indicates that when the distance between the two points is larger than the aperture diameter, the real part of the structure function becomes saturated (as shown in Fig. 2).

Similarly, the log-intensity structure function due to the imaginary part of the ARISP is

$D_{\ln I, \operatorname{Im}}(\rho)=32 \pi^{2} \eta^{2} \int_{0}^{L} \mathrm{~d} x \int_{0}^{\infty}$

$\Phi_{n, \operatorname{Im}}(\kappa) \cos ^{2}(\theta)\left[1-J_{0}(\kappa \rho)\right]$

$\left[\frac{2 J_{1}\left(\frac{D_{\mathrm{r}} \kappa x}{2 L}\right)}{D_{\mathrm{r}} \kappa x / 2 L}\right]^{2}\left[\frac{2 J_{1}\left(\frac{D_{\mathrm{t}} \kappa(L-x)}{2 L}\right)}{D_{\mathrm{t}} \kappa(L-x) / 2 L}\right]^{2} \kappa \mathrm{d} \kappa$.

As was the case with Eqs. (11) and (12), the following simplified relationships can be derived through further derivation steps:

$D_{\ln I, \operatorname{Im}}(\rho)=8.5 C_{n, \operatorname{Im}}^{2} \eta^{2} L \rho^{5 / 3}\left(D_{\mathrm{t}}\left(\right.\right.$ or $\left.\left.D_{\mathrm{r}}\right)<\rho \ll L_{0}\right)$,

$D_{\ln I, \operatorname{Im}}(\rho)=2.95 C_{n, \operatorname{Im}}^{2} \eta^{2} L \rho^{7 / 6} L_{0}^{1 / 2}\left(\rho \sim<L_{0}\right)$.

Here, the relationship corresponding to the case in which $\rho$ is less than $D_{\mathrm{t}}$ (or $D_{\mathrm{r}}$ ) is not given because under this condition the contribution of $C_{n, \operatorname{Im}}^{2}$ to the structure function is much lower than that of $C_{n, \operatorname{Re}}^{2}$ (see Fig. 2).

The results obtained from the numerical integration of Eqs. (11) and (13) with the same parameters used in Fig. 1 are plotted in Fig. 2. The solid line is the contribution of $C_{n, \mathrm{Im}}^{2}$ to the structure function, the dashed line is contribution of $C_{n, \mathrm{Re}}^{2}$ and the line with circular markers is the sum of the contributions. According to Eqs. (1) and (10), $D_{\ln I}(\rho)=$ $D_{\ln I, \operatorname{Im}}(\rho)+D_{\ln I, \operatorname{Re}}(\rho)$. When $\rho$ is relatively small, $D_{\ln I, \operatorname{Re}}$ increases with increasing $\rho$ and becomes saturated when 


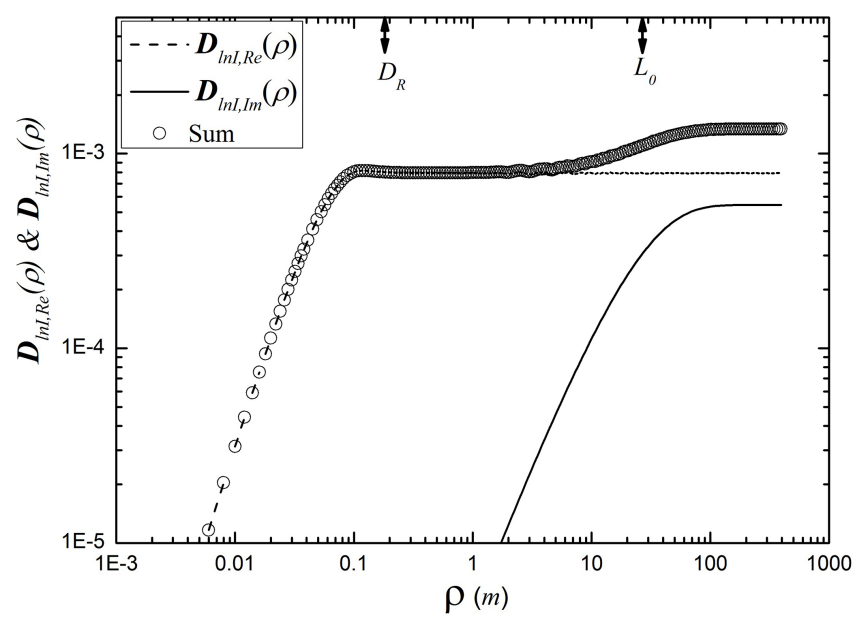

Figure 2. The temporal structure functions $\left(D_{\ln I, \operatorname{Re}}(\rho)\right.$ and $\left.D_{\ln I, \operatorname{Im}}(\rho)\right)$ of the logarithmic light intensity fluctuations due to the real and imaginary parts of the ARISP, calculated with the same parameters used in Fig. 1. The two arrows in the figure denote the values of the diameter of the transmitter (or receiver) and the outer scale to assist in comparison with different scales.

the value of $\rho$ reaches that of the LAS aperture diameter. The contribution of $C_{n, \operatorname{Im}}^{2}$ to the structure function is much smaller than that of $C_{n, \operatorname{Re}}^{2}$ when $\rho$ is less than $D_{\mathrm{t}}$ (or $D_{\mathrm{r}}$ ). When $\rho$ is larger than the aperture diameter and much smaller than the outer scale, $D_{\ln I, \operatorname{Im}}$ depends only on $C_{n, \mathrm{Im}}^{2}$, as expressed in Eq. (14a). However, the uncertainty in calculating $D_{\ln I, \text { Im }}$ is relatively large because the contribution from $C_{n, \mathrm{Re}}^{2}$ dominates when $\rho$ is smaller than $L_{0}$. When $\rho$ is close to $L_{0}$, the contribution from $C_{n, \operatorname{Im}}^{2}$ is relatively large and can be easily identified. Therefore, to reduce the noise, a proper range of $\rho$ should be selected ( $\rho$ should be close to $L_{0}$ but less than $L_{0}$ ), and Eq. (14b) can be used to determine $C_{n, \operatorname{Im}}^{2}$.

The goal of this study is to calculate the log-intensity structure function due to the imaginary part of the refractive index from the LAS measurements. Based on the previous spectral analysis, when $\rho$ is larger than the aperture diameter, the contribution of $C_{n, \mathrm{Re}}^{2}$ to the structure function becomes saturated and is twice as large as the log-intensity variance due to $C_{n, \operatorname{Re}}^{2}$ (a comparison of Eqs. (5) and (12c) gives $\left.D_{\ln I, \operatorname{Re}}(\rho)=2 \sigma_{\ln I, \operatorname{Re}}^{2}\right)$. Therefore, the contribution of $C_{n, \mathrm{Im}}^{2}$ to the structure function can be derived by subtracting twice the log-intensity variance due to $C_{n, \mathrm{Re}}^{2}$ from the measured value. That is, $D_{\ln I, \operatorname{Im}}(\rho)=D_{\ln I}(\rho)-D_{\ln I, \operatorname{Re}}(\rho)=$ $D_{\ln I, \operatorname{Im}}(\rho)-2 \sigma_{\ln I, \operatorname{Re}}^{2}(\rho)$, when $\rho$ is larger than $D_{\mathrm{t}}\left(\right.$ or $\left.D_{\mathrm{r}}\right)$. This relationship is used to calculate the imaginary part of the structure function.

In practice, the time series of measured signals is used to calculate the structure functions. According to Taylor's hypothesis, i.e. $\rho=v \tau$, Eq. (14b) can be alternatively written as

$D_{\ln I, \operatorname{Im}}(v \tau)=\gamma_{\ln I, \operatorname{Im}} \tau^{7 / 6}\left(\tau \sim<L_{0} / v\right)$,

where

$\gamma_{\mathrm{ln} I, \operatorname{Im}}=2.95 C_{n, \operatorname{Im}}^{2} \eta^{2} L v^{7 / 6} L_{0}^{1 / 2}\left(\tau \sim<L_{0} / v\right)$.

Combining Eqs. (16) and (8), we obtain

$C_{n, \operatorname{Im}}^{2}=\frac{1}{2.95} \frac{\gamma_{\ln I, \operatorname{Im}}^{10 / 7}}{\left(\sigma_{\ln I, \operatorname{Im}}^{2}\right)^{3 / 7} \eta^{2} L v^{5 / 3}}$

$L_{0}=\left(\sigma_{\ln I, \operatorname{Im}}^{2} / \gamma_{\ln I, \operatorname{Im}}\right)^{6 / 7} v$.

On the right-hand side of Eqs. (17) and (18), all of the variables are known or can be derived from LAS measurements. Therefore, Eqs. (17) and (18) can be used to calculate the imaginary part of the ARISP and the outer scale of turbulence.

\section{Experiment and data processing}

In this section, a description of the experiments and the data processing steps, which utilise the theory presented above, will be provided.

\subsection{Experimental description}

The light propagation experiments were conducted on the campus of the University of Science and Technology of China (USTC). Figure 3a shows the southern area of the city of Hefei, and Fig. $3 \mathrm{~b}$ shows the experimental site on the USTC campus. The experimental site is representative of a typical urban underlying surface. The campus is surrounded by urban roads with heavy traffic. Two main roads are located to the west and north, and a viaduct runs over the western road. Four-storey buildings and trees occupy most of the campus, which collectively have a mean height of $15 \mathrm{~m}$ (a plane with a height of $15 \mathrm{~m}$ is considered to be the reference plane). The experiments were carried out between a $55 \mathrm{~m}$ high building (symbol A in Fig. 3b) and a $62 \mathrm{~m}$ high building (symbol B in Fig. 3b), which are located at the southernmost and northernmost points of the campus and are separated by a distance of $960 \mathrm{~m}$. The transmitter was located at the southern building, and the receiver was located at the northern building, with the laser path pointing in the southnorth direction. The measurements were performed on the 12th floor of the two buildings, at a height of $24.5 \mathrm{~m}$ above the reference plane. The signal measured by a large-aperture scintillometer has a larger weight on the middle part of the propagating path (Wang et al., 1978), which is high enough to be taken as meeting the isotropy assumption (Mårtensson et al., 2006). For typical LAS measurements, the height is a very important physical quantity and should be carefully measured and calibrated (Evans and De Bruin, 2011). However, quantitative heat flux analyses were not required for this 

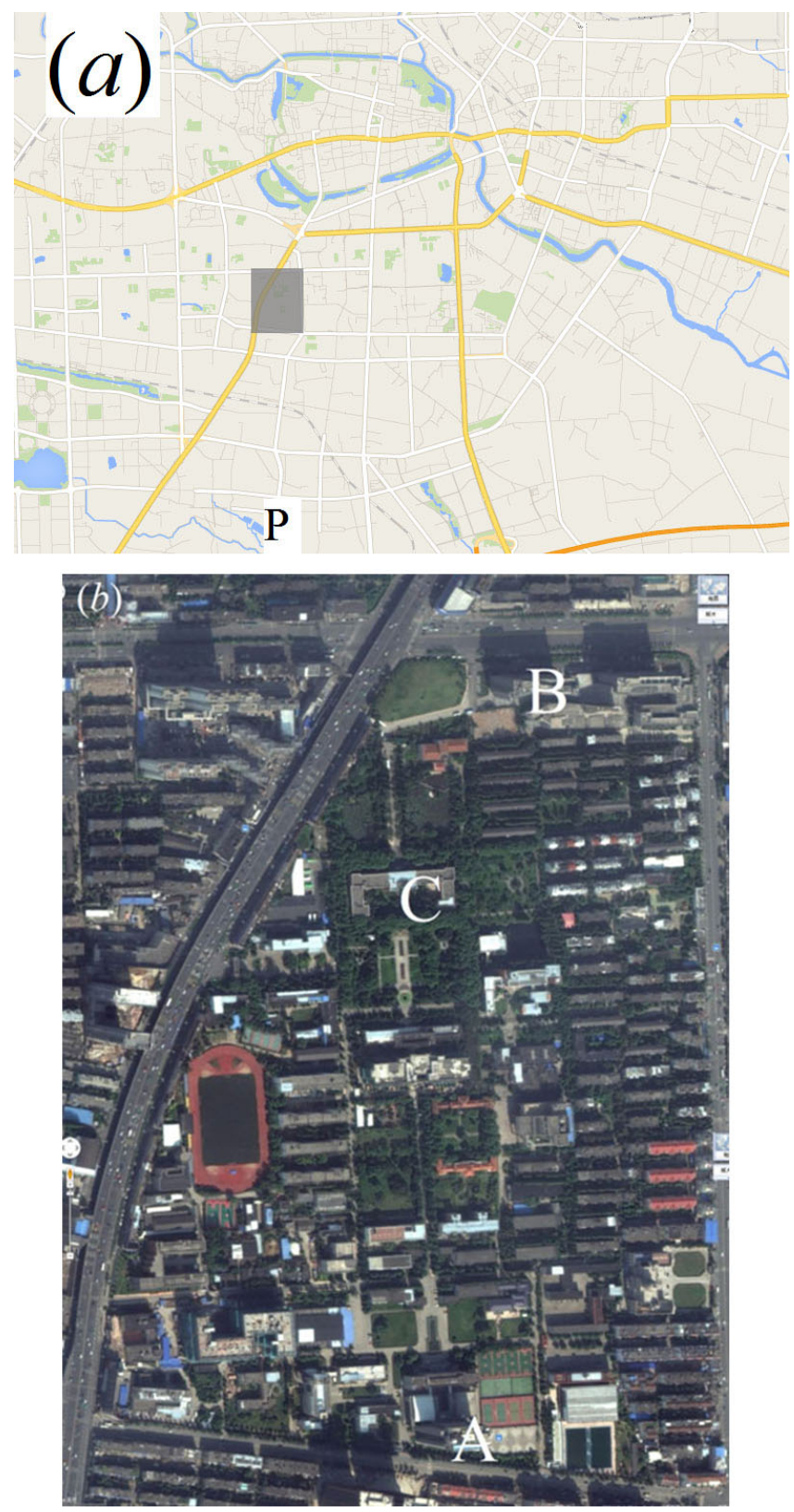

Figure 3. Photographs of the measurement site. (a) Map of the city of Hefei and (b) expanded view of the measurement site on the USTC campus, which is marked with the rectangle in (a). Point $\mathrm{P}$ in (a) indicates the site from which visibility measurements were obtained. Points A and B in (b) show the locations of the transmitter and receiver, respectively. Point $\mathrm{C}$ in (b) marks the meteorological tower position. There are four heavy-traffic roads surrounding the measurement site. Figures $3 a$ and $b^{\complement}$ Google.

study; therefore, the measurement heights reported are simply referenced to the reference plane representing the urban canopy layer.

A high meteorological tower (symbol C in Fig. 3b), whose top is $18 \mathrm{~m}$ above the reference plane, is located near the light path. Sensors for wind speed, wind direction, temperature and humidity were mounted at three levels on the tower, the uppermost level of which is at the top of the tower. The meteorological data were sampled every minute, averaged and saved every $20 \mathrm{~min}$. These measurements were used to derive the stability near the surface.

The LAS is a copy of the instrument conceived by Wang et al. (1978) and was built at USTC. The transmitting and receiving aperture diameters of the LAS used for these measurements are $0.18 \mathrm{~m}$. The light source is a light-emitting diode (LED) modulated at $116 \mathrm{kHz}$ with a wavelength of $0.620 \mu \mathrm{m}$. This wavelength is only weakly absorbed by $\mathrm{O}_{3}$; therefore, the observed absorption is primarily due to aerosols (Brion et al., 1998; Lou et al., 2014; Nebuloni, 2005). A transmitter lens converges the emitted light, which propagates over $960 \mathrm{~m}$ to the receiver.

A photo detector located at the focus of the receiving lens converts light intensities to electrical signals, which can be demodulated and amplified by an amplifier. The bandwidth of the amplifier is between 0.001 and $250 \mathrm{~Hz}$ and the output signal is sampled at a frequency of $500 \mathrm{~Hz}$. The data files are saved in 20 min intervals.

There are seven visibility measurement sites near the experimental field, which collect visibility data every $30 \mathrm{~min}$. The measurements from these sites agree well with each other, which means that the visibility at our experimental site can be represented by data obtained at these sites. In this study, we used visibility measurements obtained at a height of $6 \mathrm{~m}$ acquired from the nearest site, which is approximately $3 \mathrm{~km}$ away from our experimental site and is marked with the symbol P in Fig. 3a.

\subsection{Data analysis method and examples}

According to the theory presented in Sect. 2, the real and imaginary parts of the ARISP, the transverse wind speed and the turbulence outer scale can be retrieved and calculated from LAS measurements.

As an example, the results of the LAS observations obtained at $20 \mathrm{~min}$ intervals starting on 15 January 2014, 08:30 LT, are presented. The LAS observations were collected at $24.5 \mathrm{~m}$ above the reference plane. During the acquisition, the wind speed was $1.1 \mathrm{~m} \mathrm{~s}^{-1}$, the wind direction was $86^{\circ}$ at $18 \mathrm{~m}$ and the visibility was $6.0 \mathrm{~km}$. The calculated value of $L_{\mathrm{MO}}=-11 \mathrm{~m}$ ( $L_{\mathrm{MO}}$ is the Obukhov length) indicates that the atmosphere in the surface layer was unstably stratified.

The spectral analysis technique is used to calculate the variance at low and high frequencies. The spectrum is calculated via fast Fourier transform and plotted in Fig. 4 for the 20 min series. In Fig. 4, the spectral density of the plateau region at a frequency of approximately $1 \mathrm{~Hz}$ is $\mathrm{WP}_{\mathrm{Ln} I, \operatorname{Re}}=$ $6.3 \times 10^{-5}$. Spectral densities higher than $\mathrm{WP}_{\mathrm{Ln} I, \operatorname{Re}}$ are contributed by the imaginary part of the ARISP and are found in the lower-frequency region, whereas those lower than 
$\mathrm{WP}_{\mathrm{Ln} I, \operatorname{Re}}$ are contributed by the real part. With the measured spectrum, the spectral densities at high and low frequencies can be integrated to yield the variances of log-intensity of $4.07 \times 10^{-4}$ and $2.49 \times 10^{-4}$, respectively.

Using Eqs. (6) and (7), $C_{n, \operatorname{Re}}^{2}$ can be calculated to be $9.5 \times 10^{-15} \mathrm{~m}^{-2 / 3}$, and the transverse wind speed is $1.3 \mathrm{~m} \mathrm{~s}^{-1}$. Although there are several methods for retrieving the wind speed using a LAS (van Dinther et al., 2013), based on our results, Eq. (7) is simpler and more stable than the other approaches. To ensure the reliability of our LAS experiments, additional experiments were conducted to compare the results of $C_{n, \mathrm{Re}}^{2}$ and the transverse wind speeds obtained using other methods. Good agreement was found between the various methods employed (not shown here).

After calculating the variance at high frequencies, the structure function due to the imaginary part of the refractive index could be obtained by subtracting two times the logintensity variance due to $C_{n, \mathrm{Re}}^{2}$ from the measured refractive index; this result is shown as the hollow circles in Fig. 5. It can be observed that when the delay time is close to $1 \mathrm{~s}$, the calculated structure function exhibits very large variations. The subtraction results in large variations because the contribution due to the imaginary part of the refractive index is nearly of the same order as that of the real part. When the delay time is greater than $10 \mathrm{~s}$, the structure function follows a $7 / 6$ power law dependence, as suggested by Eq. (14b). Thus, the values of $C_{n, \mathrm{Im}}^{2}=4.0 \times 10^{-24} \mathrm{~m}^{-2 / 3}$ and $L_{0}=27.1 \mathrm{~m}$ can be calculated from Eqs. (16) and (17). Using these parameters, the structure function can be calculated from Eq. (15) and is presented as the solid line in Fig. 5. Additionally, the structure function calculated using Eq. (14b) is shown as the dashed line. As shown in Fig. 5, the simplified relationship given by Eq. (14b) predicts the structure function well when $\rho$ is within the range of 5.5-13.4 $\mathrm{m}$ (corresponding to 5$13 \mathrm{~s}$ ). The range of the $\rho$ value based on the power range is averaged from data acquired over several days to calculate the coefficient $\gamma$ in Eq. (15).

After the parameters $C_{n, \operatorname{Re}}^{2}, C_{n, \operatorname{Im}}^{2}, L_{0}$ and $v$ are calculated, the spectral curves described by Eqs. (2) and (3) can be obtained. These curves are shown as the dashed and solid line in Fig. 4, respectively. The comparison between the logintensity fluctuation power spectrum and the theoretical prediction shows that the measurements agree well with the prediction. However, at high frequencies, the measurements are higher than the theoretical predications, which may be due to measurement noise.

\section{Results}

As a case study, diurnal variations of the real and imaginary parts of the ARISP, as well as the visibility, wind speed and wind direction observed during 15-16 January 2014 are presented. The LAS observations were performed at a height of $24.5 \mathrm{~m}$. The temporal spectrum and structure function of

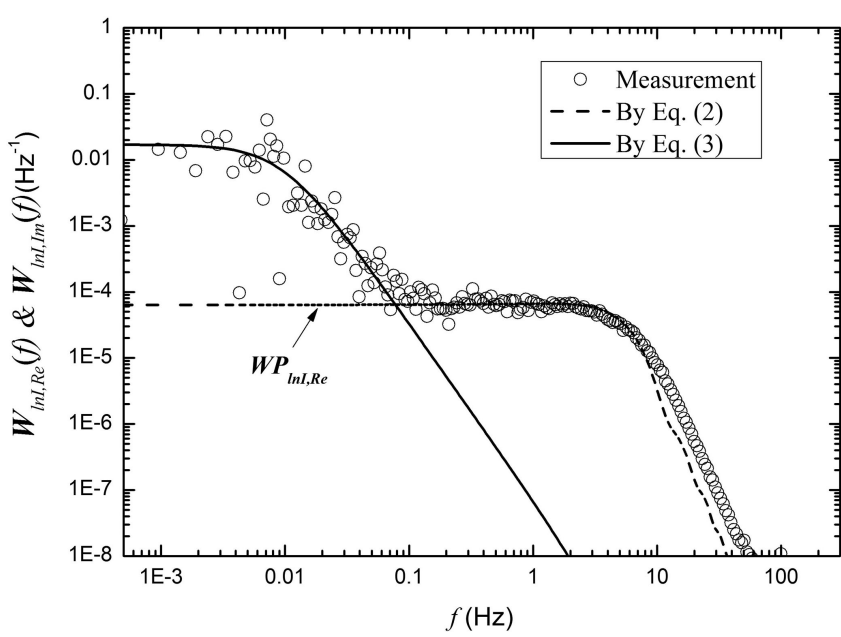

Figure 4. LAS observation at 08:30 LT on 15 January 2014 used to derive spectral parameters. A theoretical calculation is shown for comparison. The temporal spectral densities $\left(W_{\ln I, \operatorname{Re}}(f)\right.$ and $\left.W_{\ln I, \operatorname{Im}}(f)\right)$ of the logarithmic light intensity fluctuations (circles) and the theoretical curves associated with the real and imaginary parts of the ARISP (solid line and dashed line, respectively) are plotted. $\mathrm{WP}_{\ln I, \mathrm{Re}}$ refers to the spectral density of the plateau region described by Eq. (4) in the text.

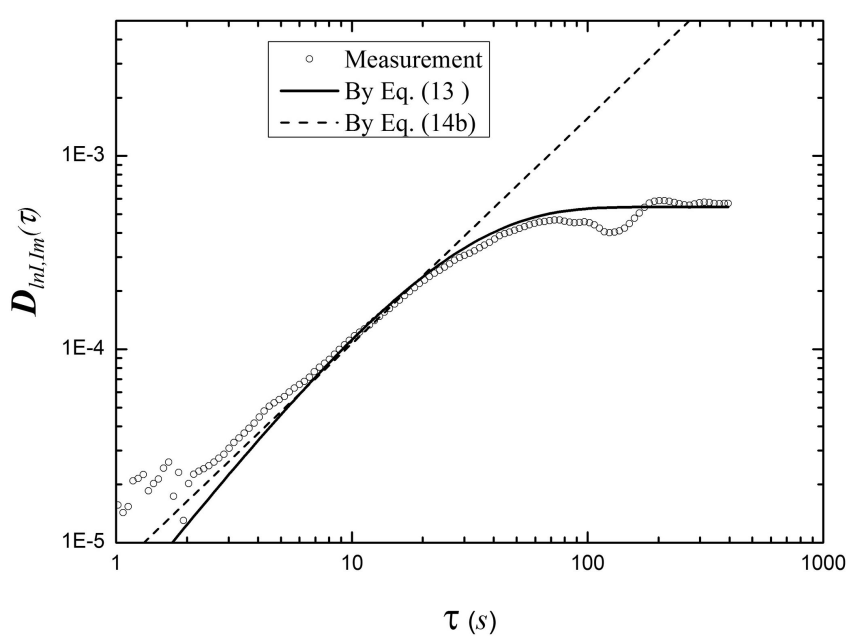

Figure 5. LAS observation (obtained at the same time as that shown in Fig. 4) used to derive the imaginary part of the ARISP and comparison with the theoretical results. The structure function of the logarithmic light intensity fluctuations caused by the imaginary part of the refractive index (circles) and the theoretical curves (solid line and dash line) are plotted.

the logarithmic light intensity acquired during these two days are shown in Figs. 6a and b, respectively. Figure 6a clearly shows that although the high-frequency part of the temporal spectrum $(\log (f)>-2)$ has a pronounced diurnal cycle with a large value at noon, this is not the case for the lowfrequency part. In Fig. 6b, the structure function of the logarithmic light intensity has a pronounced diurnal cycle over 
the entire scale range, with a large value at noon and small value during night-time.

Based on the theoretical framework presented in Sect. 2, the derived real and imaginary parts of the ARISP are shown in Figs. 7a and b. The visibilities as well as the wind speed and wind direction are also shown in Fig. $7 \mathrm{c}$ and d, respectively. Figure 7a shows that the real part of the ARISP exhibits a typical diurnal characteristic of the atmospheric boundary layer turbulence (Stull, 1988). The real part of the ARISP increases gradually after sunrise, then reaches its maximum at noon and reaches its minimum after sunset. However, the temporal evolution of the imaginary part of the ARISP displays a different pattern compared with the real part. The imaginary part of the ARISP reached a maximum at 09:00 LT on 15 January 2014, and again at 12:00 LT the next day. However, large variations in the imaginary part of the ARISP were also observed during other periods of the day. Three such large variations are labelled A, B and $\mathrm{C}$ in Fig. 7b. Figure 7c shows that the visibility exceeded approximately $8 \mathrm{~km}$ during the daytime and was less than $8 \mathrm{~km}$ during night-time. The relative humidity during the two days was less than $60 \%$; thus, the variation in visibility is mainly caused by near-surface aerosol variations associated with aerosol sources and vertical transport. During these two days, minimal near-surface visibility typically occurred at approximately 06:00 LT.

Figure $7 \mathrm{~d}$ shows weak diurnal variations in both wind speed and wind direction. The prevailing direction of the wind throughout the two days was between east and southeast, with the exception of a large variation in the wind direction after 12:00 LT on 16 January 2014. $C_{n, \mathrm{Im}}^{2}$ began to increase gradually at 06:00 LT on 15 January 2014 and reached a maximum at 09:00 LT. Figures 7a and $b$ show that the increases in $C_{n, \mathrm{Im}}^{2}$ and $C_{n, \mathrm{Re}}^{2}$ began at nearly the same time (this onset time is earlier than sunrise, which occurs at 07:00 LT in winter). Figure $7 \mathrm{~d}$ shows that the wind speed increased from 06:00 to 09:00 LT on 15 January 2014. It is known that turbulence is associated with not only convective turbulence but also wind shear. The early stage of increase observed for $C_{n, \mathrm{Im}}^{2}$ and $C_{n, \mathrm{Re}}^{2}$ appears to be caused by shear-induced turbulence in the urban surface layer. Naturally, the convective turbulence was enhanced after sunrise, which led to a quick increase in $C_{n, \mathrm{Im}}^{2}$ and $C_{n, \mathrm{Re}}^{2}$ in the early morning. A similar pattern was also observed the following morning, indicating that, as expected, turbulence controls $C_{n, \mathrm{Im}}^{2}$. However, after reaching a maximum at 09:00 LT on 15 January $2014, C_{n, \mathrm{Im}}^{2}$ decreased gradually while $C_{n, \operatorname{Re}}^{2}$ continued to increase. This situation can perhaps be explained by the variation in the visibility, which also continued to increase after 09:00 LT, implying a decreasing aerosol concentration. However, the case for the late morning of the next day is different, during which both $C_{n, \mathrm{Im}}^{2}$ and the visibility continued to increase from 09:00 to 12:00 LT. There are two possible reasons for this behaviour, one of which is that $C_{n, \mathrm{Im}}^{2}$ was dominantly controlled by turbulence during that period. For example, at point B, $C_{n, \mathrm{Im}}^{2}$ increased simultaneously with $C_{n, \mathrm{Re}}^{2}$. Another possible explanation is that $C_{n, \text { Im }}^{2}$ was influenced by a local emission of aerosols whose variation could not be detected by the visibility measurement (because the LAS measurement site is $3 \mathrm{~km}$ away from the visibility measurement site). The times $\mathrm{A}$ and $\mathrm{C}$ both fall within the local traffic rush hour, which is a strong source of local aerosol production. The peak values at times $\mathrm{A}$ and $\mathrm{C}$ suggest that $C_{n, \mathrm{Im}}^{2}$ are also dependent on aerosol distribution.

\section{Conclusions and discussion}

According to the theory of light propagation, fluctuations of logarithmic light intensity depend on both the real and the imaginary parts of the ARISP. This study focused on how to obtain the imaginary parts of the ARISP from scintillation measurements. Based on the assumption that the von Karman spectrum can be used to describe the spectrum of the imaginary part of the refractive index, the expressions of the $\log$-intensity variance and structure function were derived, and both of them include the imaginary part of the ARISP. As expressed by Eqs. (8) and (14b), these two relationships provide a method for obtaining the imaginary parts of the ARISP as well as the outer scale of turbulence from LAS measurements. The good agreement observed in this study between the measured and calculated spectra of the logarithmic light intensity and the imaginary part of structure function suggests that this method is reasonable and applicable. Although the real part of the ARISP has been previously used to measure the sensitive and the latent heat fluxes by using an LAS, deriving the imaginary part from an LAS has not been sufficiently investigated before. Therefore, the method proposed in this study can extend the application of an LAS to urban environmental monitoring because the derived imaginary parts of the ARISP include information pertaining to aerosols whose concentration is much higher in many cities than in rural areas.

Experiments of light propagation in the urban surface layer were conducted. Because the wavelength of the light source was $0.62 \mu \mathrm{m}$, the light attenuation was primarily caused by aerosols. The imaginary part of the ARISP was calculated according to our new method. The diurnal variation of the imaginary part of the ARISP is different from that of the real part. There is evidence that the imaginary part of the ARISP is associated with both the turbulence intensity and the aerosol concentration. Furthermore, the scintillation corresponding to the real part of the ARISP includes two contributions: the fluctuation of the refractive index caused by turbulence and the forward scatter caused by the aerosol particles. The scintillation corresponding to the imaginary part of the ARISP also includes two contributions: the absorption of aerosols and the scattering caused by the aerosol particles. The experimental results show that the diurnal variation 

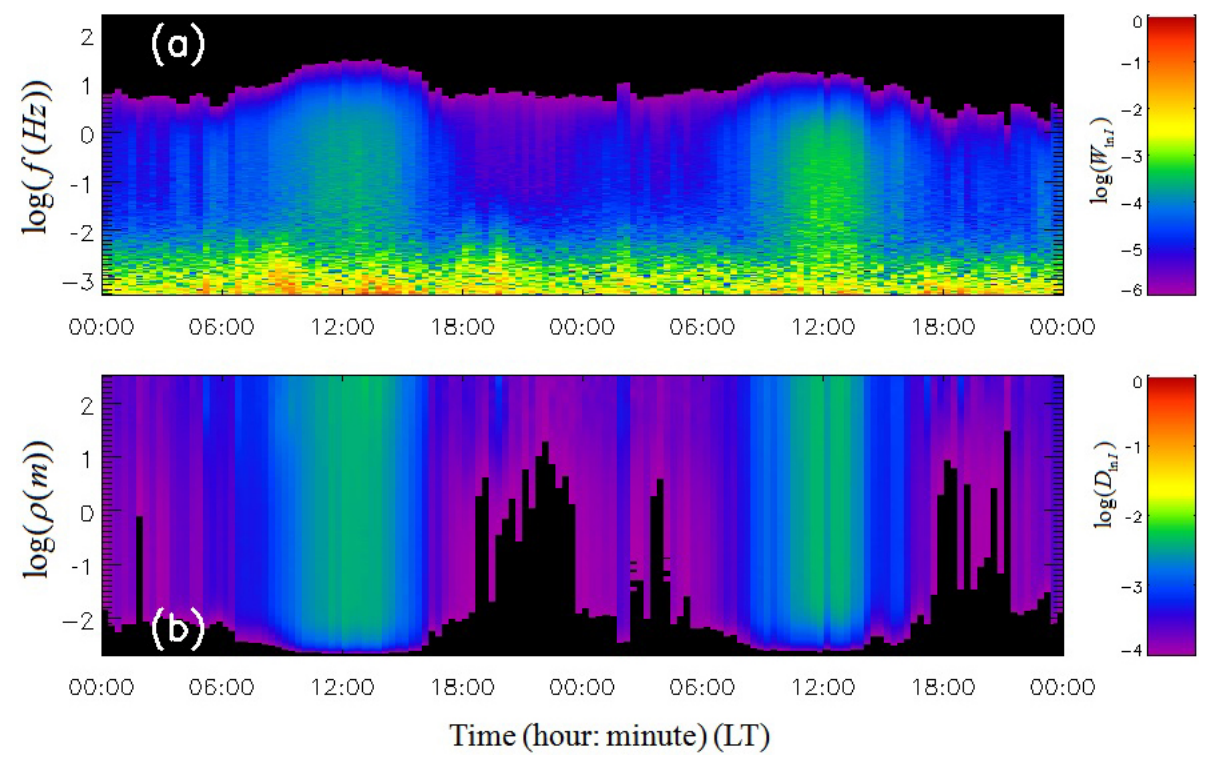

Figure 6. The evolution of the (a) temporal spectral density and (b) structure function of the logarithmic light intensity fluctuations observed during 15-16 January 2014. In panel (a), the two-dimensional colour of the contour denotes the logarithm of the temporal spectral density of the logarithmic light intensity fluctuations according to the colour bar shown on the right. In panel (b), the two-dimensional colour contour denotes the logarithm of the structure function of the logarithmic light intensity fluctuations according to the colour bar shown on the right. The black areas indicate values that are below the minimum of the colour scale.

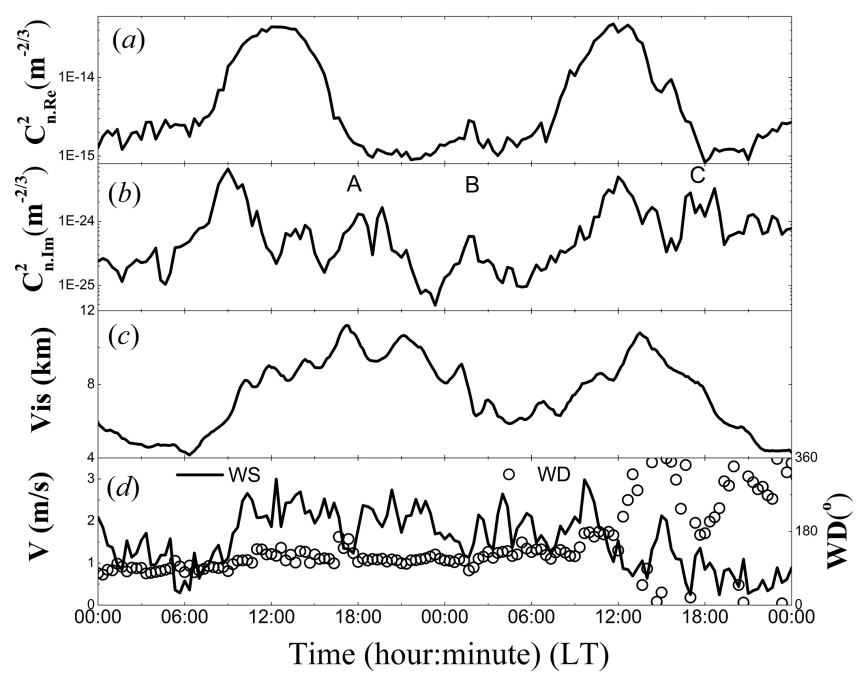

Figure 7. Temporal variations of the (a) real part of the ARISP, (b) imaginary part of the ARISP, (c) visibility and (d) wind speed and direction observed during 15-16 January 2014. The points labelled $\mathrm{A}, \mathrm{B}$ and $\mathrm{C}$ in panel (b) highlight the three periods with large values for the imaginary part of the ARISP other than the two daily maxima. Details can be found in the text.

of the real part of the ARISP exhibits the typical pattern of the diurnal variation of turbulence intensity, suggesting that the real part of the ARISP is dominantly controlled by turbulence, whereas the scattering effect of aerosol particles is negligibly small. However, the information included in the imaginary part of the ARISP appears to be more complex. The absorption is dependent not only on the aerosol concentration but also on chemical composition. Additionally, the scattering effect is influenced not only by the aerosol concentration but also by the aerosol particle size distribution and the shape of the aerosol particles. Presently, it is not clear to what extent chemical composition, size distribution and particle shape can affect the imaginary part of the ARISP compared with the aerosol concentration. If the contribution of these variables is very small, the imaginary part of the ARISP is simply dependent on the aerosol concentration. However, if the contribution of these variables is relatively large, and their contributions are distinguishable, more information regarding the aerosols can be obtained. Although these problems need to be investigated further, the method proposed in this study provides a starting point.

The extinction in the transport path is caused by absorption and scattering by aerosols. The imaginary part of the ARISP represents the logarithmic light intensity fluctuations caused by the fluctuations of the extinction, which in turn should be mainly induced by fluctuations in the aerosol concentration, assuming that other conditions such as chemical composition, size distribution and shape are unchanged. It is clear that fluctuations in the aerosol concentration are associated with the aerosol concentration itself and the intensity of turbulent motion. Consequently, our experiments showed that the imaginary part of the ARISP is influenced by turbulence and changes in the aerosol concentration. With respect to measuring the extinction of the atmosphere, long-path ab- 
sorption spectroscopy can be used to obtain the attenuation in a narrow band and to derive the gas concentration (Fiddler et al., 2009). The advantage of the spectroscopy is to attain the narrow spectral information. The LAS method can be used to measure the high- and low-frequency fluctuations of light intensity, and more information - including atmospheric temperature, crosswind, aerosol absorption and other turbulence characteristics - can be obtained. If the employed spectroscopy can sample the attenuated light at a high rate at every narrow band, then additional aerosol parameters can be retrieved using the current method. The method developed in this study has the potential to allow for the retrieval of more information from aerosols.

Previous observations have shown that aerosol concentration fluctuations follow the characteristics of scalars (Mårtensson et al., 2006; Vogt et al., 2011), implying that the imaginary part of the ARISP might contain aerosol transport information similar to the relationship between the temperature structure parameter and the sensible heat flux. Our measurement site is in the city of Hefei, which has a population of approximately 3500000 and hosts more than 500000 vehicles. For cities similar to Hefei, e.g. Helsinki, Finland, vehicles are a primary pollution source (Järvi et al., 2009). The temporal variation of the imaginary part of the ARISP is well correlated with the variation of the aerosol flux (Ripamonti et al., 2013). These pieces of evidence suggest that the imaginary part of the ARISP can be used to measure the aerosol flux via LAS. Determining whether the imaginary part of the ARISP represents aerosol flux or concentration is an issue that should be investigated further.

Based on the theoretical analysis discussed herein, it can be expected that the method presented in this study can also be applied to measure the imaginary part of the ARISP caused by atmospheric trace gases if the wavelength of light used is selected to be within the corresponding gas absorption region.

Acknowledgements. This study was supported by the National Natural Science Foundation of China (41475012, 41230419, 91337213 and 41075041), Anhui Provincial Natural Science Foundation of China (1208085MF100) and the Jiangsu Provincial Collaborative Innovation Center of Climate Change. Thanks are also given to Guijian Liu, Yefei Yuan, Yu Wang, Guoshen Liu, Chune Shi and Renjun Zhou for their help in collecting data and preparing the experiments.

Edited by: X. Liu

\section{References}

Andreas, E. L.: Two-wavelength method of measuring pathaveraged turbulent surface heat fluxes, J. Atmos. Ocean Tech., 6, 280-292, 1989.
Andrews, L. C. and Phillips, R. L.: Laser beam propagation through random media, SPIE, Bellingham, Washington, USA, 782 pp., 2005.

Brion, J., Chakir, A., Charbonnier, J., Daumont, D., Parisse, C., and Malicet, J.: Absorption spectra measurements for the ozone molecule in the 350-830 nm region, J. Atmos. Chem., 30, 291299, doi:10.1023/a:1006036924364, 1998.

Clifford, S. F.: Temporal-frequency spectra for a spherical wave propagating throught atmospheric turbulence, J. Opt. Soc. Am., 61, 1285-1292, 1971.

Consortini, A., Ronchi, L., and Stefanut.L: Investigation of atmospheric turbulence by narrow laser beams, Appl. Opt., 9, 25432547, doi:10.1364/ao.9.002543, 1970.

De Bruin, H. A. R. and Evans, J. G.: Long path scintillometry: a brief review, in: Remote Sensing and Hydrology, edited by: Neale, C. M. U. and Cosh, M. H., IAHS Publication, Wallingford, UK, 180-183, 2012.

Ding, Y. H. and Liu, Y. J.: Analysis of long-term variations of fog and haze in China in recent 50 years and their relations with atmospheric humidity, Science China: Earth Sciences, 57, 36-46, 2014.

Evans, J. G. and De Bruin, H. A. R.: The Effective Height of a TwoWavelength Scintillometer System, Bound-Lay. Meteorol., 141, 165-177, doi:10.1007/s10546-011-9634-0, 2011.

Fiddler, M. N., Begashaw, I., Mickens, M. A., Collingwood, M. S., Assefa, Z., and Bililign, S.: Laser Spectroscopy for Atmospheric and Environmental Sensing, Sensors, 9, 10447-10512, doi:10.3390/s91210447, 2009.

Filho, F. C. M., Jayasuriya, D. A. R., Cole, R. S., and Helmis, C. G.: Spectral density of millimeter wave amplitude scintillations in an absorption region, IEEE T. Antenn. Propag., 31, 672-676, 1983.

Ishimaru, A.: Wave propagation and scattering in random media, Oxford University Press New York, 574 pp., 1997.

Järvi, L., Rannik, Ü., Mammarella, I., Sogachev, A., Aalto, P. P., Keronen, P., Siivola, E., Kulmala, M., and Vesala, T.: Annual particle flux observations over a heterogeneous urban area, Atmos. Chem. Phys., 9, 7847-7856, doi:10.5194/acp-9-7847-2009, 2009.

Lou, S., Liao, H., and Zhu, B.: Impacts of aerosols on surface-layer ozone concentrations in China through heterogeneous reactions and changes in photolysis rates, Atmos. Environ., 85, 123-138, doi:10.1016/j.atmosenv.2013.12.004, 2014.

Mårtensson, E. M., Nilsson, E. D., Buzorius, G., and Johansson, C.: Eddy covariance measurements and parameterisation of traffic related particle emissions in an urban environment, Atmos. Chem. Phys., 6, 769-785, doi:10.5194/acp-6-769-2006, 2006.

Nebuloni, R.: Empirical relationships between extinction coefficient and visibility in fog, Appl. Opt., 44, 3795-3804, doi:10.1364/ao.44.003795, 2005.

Nieveen, J. P., Green, A. E., and Kohsiek, W.: Using a large-aperture scintillometer to measure absorption and refractive index fluctuations, Bound-Lay. Meteorol., 87, 101-116, 1998.

Ott, R. H. and Thompson Jr., M. C.: Atmospheric Amplitude Spectra in an Absorption Region, IEEE T. Antenn. Propag., 16, 329332, 1978.

Rao, R.: Modern Atmospheric Optics, Science Press, Beijing, 608 pp., 2012. 
Raut, J.-C. and Chazette, P.: Vertical profiles of urban aerosol complex refractive index in the frame of ESQUIF airborne measurements, Atmos. Chem. Phys., 8, 901-919, doi:10.5194/acp-8-9012008, 2008.

Ripamonti, G., Jarvi, L., Molgaard, B., Hussein, T., Nordbo, A., and Hameri, K.: The effect of local sources on aerosol particle number size distribution, concentrations and fluxes in Helsinki, Finland, TELLUS B, 65, 19786, doi:10.3402/tellusb.v65i0.19786, 2013.

Solignac, P. A., Brut, A., Selves, J. L., Beteille, J. P., and GastelluEtchegorry, J. P.: Attenuating the Absorption Contribution on C-n2 Estimates with a Large-Aperture Scintillometer, BoundLay. Meteorol., 143, 261-283, doi:10.1007/s10546-011-9692-3, 2012.

Stull, R. B.: An Introduction to Boundary Layer Meteorology, Kluwer Academic Publishers, Boston, USA, 666 pp., 1988.

Tatarskii, V. I.: Wave Propagation in a Turbulent Medium, McGrawHill Book Company Inc., New York, 285 pp., 1961.

van Dinther, D., Hartogensis, O. K., and Moene, A. F.: Crosswinds from a Single-Aperture Scintillometer Using Spectral Techniques, J. Atmos. Ocean. Tech., 30, 3-21, doi:10.1175/jtech-d12-00069.1, 2013.
Vogt, M., Nilsson, E. D., Ahlm, L., Mårtensson, E. M., and Johansson, C.: The relationship between $0.25-2.5 \mu \mathrm{m}$ aerosol and $\mathrm{CO}_{2}$ emissions over a city, Atmos. Chem. Phys., 11, 4851-4859, doi:10.5194/acp-11-4851-2011, 2011.

Wang, T. I., Ochs, G. R., and Clifford, S. F.: Saturation-resistant optical scintillometer to measure $\mathrm{Cn}_{2}$, J. Opt. Soc. Am., 68, 334 338, 1978.

Wyngaard, J. C., Izumi, Y., and Collins, S. A.: Behavior of refractive-index-structure parameter near ground, J. Opt. Soc. Am, 61, 1646-1650, doi:10.1364/josa.61.001646, 1971.

Yuan, R., Sun, J., Luo, T., Wu, X., Wang, C., and Fu, Y.: Simulation study on light propagation in an anisotropic turbulence field of entrainment zone, Opt. Express, 22, 13427-13437, 2014.

Zhang, X., Huang, Y., Rao, R., and Wang, Z.: Retrieval of effective complex refractive index from intensive measurements of characteristics of ambient aerosols in the boundary layer, Opt Express, 21, 17849-17862, doi:10.1364/oe.21.017849, 2013. 